\title{
Clinical Application of Dynamic Gait Index-Brazilian Brief Version
}

\author{
Carlos Kazuo Taguchi ${ }^{*}$, Élida Pinto Costa ${ }^{2}$, Lucas Vieira Alves ${ }^{2}$, Larissa Karoline Santos ${ }^{2}$, \\ Erbson Rodrigues de Oliveira Silva ${ }^{3}$, Brenda Carla de Lima Araújo ${ }^{4}$, \\ Fabíola Andréa Andrade dos Santos ${ }^{5}$, Allan Robert da Silva ${ }^{6}$
}

\author{
${ }^{1}$ Audiology Departament, Federal University of Sergipe, São Cristóvão, Brazil \\ ${ }^{2}$ Federal University of Sergipe, São Cristóvão, Brazil \\ ${ }^{3}$ Education Coordinator of Sergipe State, Aracaju, Brazil \\ ${ }^{4}$ Speech Therapist and Audiology Departament, Federal University of Sergipe, São Cristóvão, Brazil \\ ${ }^{5}$ University Hospital Board, Federal University of Sergipe, São Cristóvão, Brazil \\ ${ }^{6}$ Statistical Departament, Federal University of Sergipe, São Cristóvão, Brazil \\ Email: *carlostaguchi@hotmail.com
}

How to cite this paper: Taguchi, C.K., Costa, É.P., Alves, L.V., Santos, L.K., de Oliveira Silva, E.R., de Lima Araújo, B.C., dos Santos, F.A.A. and da Silva, A.R. (2018) Clinical Application of Dynamic Gait Index-Brazilian Brief Version. Advances in Aging Research, 7, 113-118.

https://doi.org/10.4236/aar.2018.76009

Received: September 12, 2018

Accepted: October 30, 2018

Published: November 2, 2018

Copyright $\odot 2018$ by authors and Scientific Research Publishing Inc. This work is licensed under the Creative Commons Attribution International License (CC BY 4.0).

http://creativecommons.org/licenses/by/4.0/

\begin{abstract}
Introduction: In elderly, the progressive degenerations in the nervous system and vestibular system compromise the human body balance leading to a greater risk of falls. The Dynamic Gait Index is a protocol that predicts risks to falls in seniors. Objective: To analyze the results of the application of the Dynamic Gait Index (DGI)-Brazilian brief proposal and establish correlation with the original version. Methods: Study approved by the Ethics and Research Committee of Federal University of Sergipe (number 0197.0.107000.09). We analyzed 223 files of socially active elderly, female gender, aged ranged 60 to 85 years, mean $68.54( \pm 7.61)$ years since 2014 . The volunteers were evaluated by the DGI-Brazil. For the statistical analysis, the Pearson Correlation Momentum Correlation Test with Spearman Correlation was used, with $\mathrm{p} \leq 0.05$ and $r=1.0$. Results: Sixty-three (27.8\%) of the volunteers presented an altered DGI scores. The statistical analysis indicated that Task 1 and Task 2 did not contribute to decrease the total score in the test. The worst performances occurred in Tasks 3, 5 and 6 . There was founded a positive correlation between Tasks 1 and 2; 1 and 8; 3 and 4; 3 and 5; 4 and 5; 2 and 4; 2 and 7. Negative correlation occurred between Tasks 4 and 8 , and 6 was not correlated with another Task. Conclusion: The DGI-Brazilian brief version presented an excellent correlation with the original proposal and may be useful in clinical practice as a tool to evidence future risks of falls in the elderly people.
\end{abstract}




\section{Keywords}

Aged, Postural Balance, Accidental Falls, Clinical Protocols

\section{Introduction}

In elderly, the progressive degenerations in the nervous system and vestibular system compromise the human body balance leading to a greater risk of falls. The deteriorated gait compromises the capacity and functional autonomy and impacting negatively the quality of life. There are differences in gait efficiency that are related to the life cycles, it is noted that it reaches its maximum efficiency at the end of childhood and declines progressively over the years with senescence. The gait gets worse and affects functional capacity in elderly and, compromises independence, autonomy and increases the rates of falls. To evaluate the gait, the Dynamic Gait Index (DGI) [1] was suggested to document the patient's ability to change gait in response to changes in their eight tasks. The DGI was validated in Brazil [2] in 2016. It's possible to hypothesize that each task of the DGI affects the final score. The objective of this study was to analyze the results of the application of the Dynamic Gait Index-Brazilian brief version and establish correlation with the original protocol.

\section{Method}

Retrospective, descriptive and qualitative study approved by the Ethics and Research Committee of the Federal University of Sergipe (number 0197.0.107000.09). We analyzed 223 patient files with evaluations of socially active elderly, female gender, aged ranged 60 to 85 years. The base data was collected on five towns of Sergipe state on northweast of Brazil since 2014. All patients with ability to respond to the DGI tasks was included. Patients with impaired verbal comprehension, motor and locomotion difficulties, visual and auditory deficiency, degenerative neurological diseases were excluded. To evaluate balance and gait, the Dynamic Gait Index-Brazil (DGI) [2] (Table 1) was used to identify future risk of falls. This study followed three steps.

In the first stage, it was verified 63 volunteers with risk of falls and the study of possible correlations between the performance between the tasks and each one of them and the result of the general performance in the DGI-Brazil [2] was carried out. It was hypothesized that each task would contribute with greater or lesser influence on the final scores and became the protocol sensitive to identify risks of falls. From the exclusion of tasks 1; 2 and 7 were defined the DGI-Brazilian brief version composed of five (5) tasks and cut point equal or less than 11 (Table 2).

In the second step of the study, all sample data was analyzed through DGI-Brazilian brief version and the possible correlations between the two versions were established in the third step. 
Table 1. Dynamic Gait Index-Brazil ${ }^{2}$ taks.

\begin{tabular}{cc}
\hline Number & Task \\
\hline T1 & Gait level surface \\
T2 & Change in gait speed \\
T3 & Gait with horizontal head turns \\
T4 & Gait with vertical head turns \\
T5 & Gait and pivot turn \\
T6 & Step over obstacle \\
T7 & Change in gait speed \\
T8 & Rise and descend steps \\
\hline
\end{tabular}

Table 2. Dynamic Gait Index-Brazilian brief taks.

\begin{tabular}{cc}
\hline Number & Task \\
\hline T1 & Gait with horizontal head turns \\
T2 & Gait with vertical head turns \\
T3 & Gait and pivot turn \\
T4 & Step over obstacle \\
T5 & Rise and descend steps \\
\hline
\end{tabular}

For the statistical analysis it was adopted as dependent variable the response of each volunteer in the DGI, the total scores in each version and which independent variable the final average in each task. It was used the Soft R Project: 3.12 to perform the descriptive measures, the Pearson Correlation Moment Product Coefficient test ( $\mathrm{p} \leq 0.05)$, the Chi-square test and the Pearson Correlation Matrix $(r=1.0)$.

\section{Results and Discussion}

It was possible identified $63(28.0 \%)$ subjects with risks to fall with the DGI in its original version. The mean age of this sample was $68.54( \pm 7.61)$ years. The Pearson Correlation Moment Product Coefficient test between each task showed a positive correlation between $\mathrm{T} 1$ and $\mathrm{T} 2(\mathrm{r}=0.37)$; $\mathrm{T} 1$ and $\mathrm{T} 8(\mathrm{r}=0.26)$; $\mathrm{T} 3$ and $\mathrm{T} 4(\mathrm{r}=0.42)$; $\mathrm{T} 3$ and $\mathrm{T} 5(\mathrm{r}=0.25)$; $\mathrm{T} 4$ and $\mathrm{T} 5(\mathrm{r}=0.33)$; $\mathrm{T} 2$ and $\mathrm{T} 4(\mathrm{r}=0.30)$ and T2 with T7 $(r=0.20)$. There was a negative correlation between T4 with T8 $(r=-0.29$. It was no found correlations withT6. Statistical analysis indicated that T1, T2 and T7 did not contribute to decrease the total score in the test and that the worst performances occurred in Tasks 3, 5 and 6 .

These results pointed five tasks were more sensitive to identify risk of falls and allowed claim that DGI-Brazilian Brief could be apply for clinical proposals (Figure 1).

At second step of the study, the 223 patient's records were reviewed by the proposal of the Brazilian DGI-brief. Statistical date suggested11 points as cut 


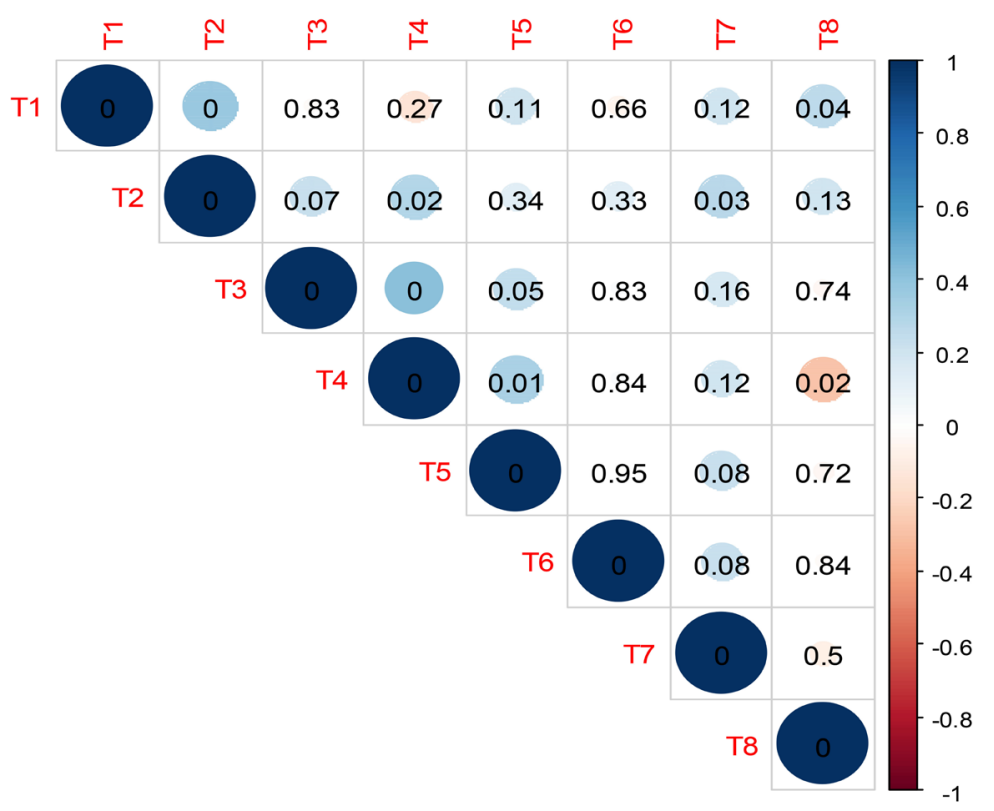

Figure 1. The Pearson Correlation Moment Product Coefficient test Matrix between each task of 63 socially active volunteers $(\mathrm{p} \leq 0.05)$. Legend: $\mathrm{T}=$ task.

score. It was possible identified $65(29.1 \%)$ volunteers with risk for falls. Fifty-seven (90.5\%) volunteers were considered within the risk parameters in the both versions of the DGI; Six (9.5\%) who were at risk in the original version came out of this condition in the brief version and eight (12.6\%) who were not at risk in the original version became in this status on the reduced version. The Chi-square test revealed no significant correlation $(\mathrm{p}=0.91)$ between the two versions, which allowed to infer that the reduced version can be used for clinical proposal but revealed a strong correlation on both version $(\mathrm{r}=1.0)$.

The positive correlations verified in the comparison of the tasks on two tools can be explained by evaluating similar functional bases, as well as the tasks that presented negative correlations, since they evaluate different attributes. One example with high correlation it was founded between tasks 3 and 4, in which the patient moves from one point to another with head movement, ranging horizontal to vertical direction. Tasks 4 and 8 were negatively correlated because they were very different activities and needed singular and greater postural control.

Mainly the results pointed that a significant percentage of the volunteers evaluated presented risks for falls, it is possible emphasize that even though they were socially active, they presented a risk for future falls. As the elderly population increases globally become urgent the development of preventive risks to falls strategies [9].

The Dynamic Gait Index-Brazil showed a high internal consistency between its tasks and could be a reliable instrument [2] [3]. There is a correlation between body balance; functional capacity, risk and prevalence for falls in patients with chronic vestibular dysfunction that pointed out a positive correlation with 
the functional capacity and balance and negative correlation with risk for falls [4]. A study [5] noticed that there was influence of the cognitive function on the final scores of the DGI, and it referred no relation between risk for falls and quality of life in socially active elderly [6].

Some studies [7] [8] showed that gait was associated with loss of strength and muscles' elasticity, postural changes, reduced mobility, poor related self-perception of health, depression, anxiety, prescription of medications, decreased vision, and order issues social and environmental issues.

This brief version agreed with other studies [9] [10] [11]. Although simple, DGI has a high predictive value for falls risk [2]. This suggestion of a brief version was disagreed a study [12]. By its clinical applicability and solid results in based scientific evidence, the results statistically indicate that the use brief instrument is agreement with other studies [13] [14] that suggested the importance of sensitive, reliable and fast applicability tools for postural control and balance evaluation.

The proposal the balance evaluation with protocols is a simplest way to verify everyone's ability to control the balance and to identify those who are likely to fall is consonant with the revealed results. The DGI is a reliability and excellent predictive protocol for risk of falls to apply on the elderly [9].

\section{Conclusion}

The DGI-Brazilian brief version presented an excellent correlation with the original proposal and may be perfectly useful in clinical to point future risks of falls in the elderly people.

\section{Conflicts of Interest}

The authors declare no conflicts of interest regarding the publication of this paper.

\section{References}

[1] Shumway-Cook, A. and Woolacott, M.H. (1995) Control of Posture and Balance. In: Shumway-Cook, A. and Woolacott, M.H., Eds., Motor Control Theory and Practical Applications, Williams \& Wilkins, Maryland, 120.

[2] Castro, S.M., Perracini, M.R. and Gananca, F.F. (2006) Versão brasileira do Dynamic Gait Index. BrazilianJournal of Otorhinolyngology, 72, 817-825. https://doi.org/10.1590/S0034-72992006000600014

[3] Peel, N.M., Kuys, S.S. and Klein, K. (2012) Gait Speed as a Measure in Geriatric Assessment in Clinical Settings: A Systematic. Review. The Journals of Gerontology, 68, 39-46. https://doi.org/10.1093/gerona/gls174

[4] Sousa, R.F., Gazzola, J.M., Ganança, M.M. and Paulino, C.A. (2011) Correlação entre equilíbrio corporal e capacidade functional de idosos com disfunções vestibulares crônicas. Brazilian Journal of Otorhinolaryngology, 77.

[5] Lenardt, M.H., Sousa, J.A., Grden, C.R.B., Betiolli, S.E., Carneiro, N.H.K. and Ribeiro, D.K.M.N. (2015) Gait Speed and Cognitive Score in Elderly Users of the Primary Care Servisse. BrazilianJournal of Nursing, 68, 851-856. 
[6] Taguchi, C.K., Teixeira, J.P., Alves, L.V., Oliveira, P.F. and Raposo, O.F.F. (2016) Quality of Life and Gait in Elderly Group. International Archives of Otorhinolaryngology, 20, 235-240.

[7] Beck, A.P. (2011) Fatores associados às quedas entre idosos praticantes de atividades físicas. Texto Contexto-Enferm, 20, 280-286. https://doi.org/10.1590/S0104-07072011000200009

[8] Denkinger, M.D., Lukas, A., Nikolaus, T. and Hauer, K. (2015) Factors Associated with Fear of Falling and Associated Activity Restriction in Community-Dwelling Older Adults: A Systematic Review. The American Journal of Geriatric Psychiatry, 23, 72-86. https://doi.org/10.1016/j.jagp.2014.03.002

[9] Alves, L.V., Taguchi, C.K., Oliveira, I.L. and Sousa, M.G.C. (2014) Avaliação de tendência à quedas em idosos de Sergipe. Rev CEFAC, 16, 1389-1396.

https://doi.org/10.1590/1982-0216201425312

[10] Taguchi, C.K., Teixeira, J.P., Costa, E.P., Freitas, L.N., Silva, A.R. and Nascimento, Y.N. (2017) Correlation between Tasks and Total Scores in Brazilian Dynamic Gait Index. Annals of 16 Congress of Otorhinolaryngology Fundation in Archives of International Otorhinolaryngology, S92-S93.

[11] Nascimento, J.S. and Tavares, D.M.S. (2016) Prevalência e fatores associados a quedas em idosos. Textocontexto-Enferm, 25.

https://doi.org/10.1590/0104-07072016000360015

[12] Matsuda, P.N., Taylor, C. and Shumway-Cook, A. (2015) Examining the Relationship between Medical Diagnoses and Patterns of Performance on the Modified Dynamic Gait Index. Journal of the American Physical Therapy Association, 95.

[13] Leal, J.B., Leal, J.B., Anjos, E.R.P., Conceição, A.J. and Cieslak, F. (2016) Instrumentos de avaliação do equilíbrio e da mobilidade funcional em idosos. Anais do Congresso brasileiro de ciências da saúde.

[14] Woellner, S.S., Araujo, A.G.S. and Martins, J.S. (2014) Protocolos de equilíbrio e quedas em idosos. Neurociências, 10, 104-117. 